\title{
Radical Polymerization Behavior of Polystyrene Macromonomers in the Presence of Inert Polystyrene
}

\author{
Kiyoharu Tsutsumi, Yasuhisa Tsukahara, ${ }^{\dagger}, *$ and Yoshio OKamoto \\ Department of Applied Chemistry, Faculty of Engineering, Nagoya University, \\ Furo-cho, Chikusa-ku, Nagoya 464-01, Japan \\ ${ }^{\dagger}$ Department of Materials Science, Kyoto Institute of Technology, \\ Matsugasaki, Sakyo-ku, Kyoto 606, Japan
}

(Received March 28, 1994)

\begin{abstract}
Radical polymerizations of polystyrene macromonomers having a methacryloyl end group with $M_{n}$ s of 2900 and 9600 were investigated in the presence of inert polystyrene (PSt) of the same molecular weight. Under homogeneous conditions, the viscosity of polymerization media was fixed with variation in macromonomer concentration ([M]) by fixing the sum of the concentrations of the macromonomer and inert PSt. The presence of the inert PSt increased both the degree of polymerization $(D P)$ of poly(macromonomer)s and polymerization rate $\left(R_{\mathrm{p}}\right)$. The observed kinetic order of $R_{\mathrm{p}}$ with respect to [M] decreased by the addition of inert PSt, but remained larger than unity. Termination rate constant $\left(k_{\mathrm{t}}\right)$ decreased by the addition of inert PSt and increase of $[\mathrm{M}]$, while the propagation rate constant $\left(k_{\mathrm{p}}\right)$ was not affected by inert PSt. $k_{\mathrm{p}}$ did not show clear [M]-dependence, irrespective of the presence of inert PSt. These results are discussed in terms of the viscosity of polymerization media, initiator efficiency and specific multibranched structure about the propagating radical.

KEY WORDS Macromonomer/Inert Polystyrene / Radical Polymerization

/ Diffusion-Control Effect / Segment Density / Viscosity of Polymerization

Solution /
\end{abstract}

Radical polymerization is strongly influenced by the viscosity of polymerization media since termination reactions of the propagating radicals are fast and easily diffusion-controlled by increase in viscosity. Thus, autoacceleration phenomenon is normally observed during the course of polymerization of conventional small monomers. ${ }^{1-4}$ On the other hand, the time-conversion curve in the radical polymerization of the macromonomer is monotonous and no autoacceleration effect is observed. ${ }^{5-9}$ The polymerization rate $\left(R_{\mathrm{p}}\right)$ and degree of polymerization $(D P)$ strongly depend on macromonomer concentration $([\mathrm{M}]) .{ }^{5-11}$ This indicates that viscosity effect does not change much during the course of polymerization but changes drastically with [M] because of the high molecular weights of macromonomers. Besides the viscosity of polymerization media, the polymerization of macromonomers is also influenced by solvent ${ }^{12}$ and aggregation or micelle formation. ${ }^{13,14}$

This paper reports radical polymerizations of the polystyrene macromonomers in the presence of inert polystyrene (PSt) under the homogeneous conditions. Inert PSt was used to hold the viscosity of polymerization media constant with variation in $[\mathrm{M}]$ by fixing the sum of the concentrations of macromonomer and inert PSt of the same molecular weight.

* To whom correspondence should be addressed. 


\section{EXPERIMENTAL}

\section{Materials}

In this study, polystyrene macromonomers having a methacryloyl end group (MA-PSt) were prepared. Scheme I shows the preparation procedure of the macromonomer and the inert PSt which has the same molecular weight with the macromonomer. Polystyryllithium living anion formed by living anionic polymerization of styrene at $-78^{\circ} \mathrm{C}$ under dried $\mathrm{N}_{2}$ atmosphere was separated into two parts. One of them was reacted with ethylene oxide and then terminated by methacryloyl chloride to prepare the macromonomer. The other was terminated by methanol to obtain inert PSt which has the same molecular weight with the macromonomer. The macromonomer and the inert

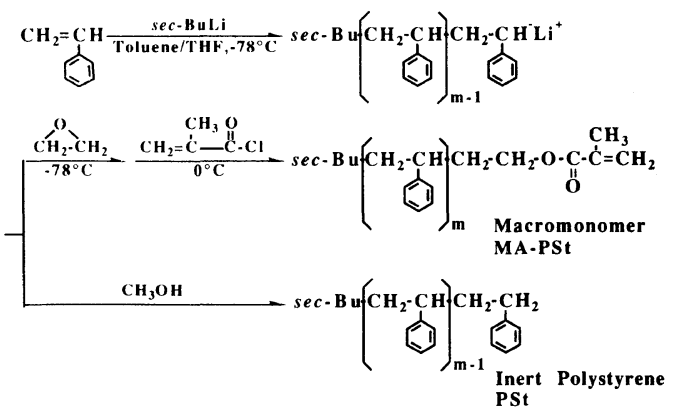

Scheme I. Preparation of polystyrene macromonomer having a methacryloyl end group (MA-PSt) and inert polystyrene (PSt).

Table I. Characteristics of polystyrene macromonomer having a methacryloyl end group (MA-PSt) and inert polystyrene (PSt)

\begin{tabular}{cccccc}
\hline Code & $M_{n}$ & $M_{w}$ & $M_{w} / M_{n}$ & \multicolumn{2}{c}{ End Functionality } \\
\cline { 3 - 6 } & & & & ${ }^{1}$ H NMR $^{\mathrm{a}}$ & M.C. $^{\mathrm{b}}$ \\
\hline MA-PSt2900 & 2900 & 3100 & 1.07 & 0.93 & 0.93 \\
PSt2900 & 2900 & 3100 & 1.07 & - & - \\
$\begin{array}{c}\text { MA-PSt9600 } \\
\text { PSt9400 }\end{array}$ & 9600 & 9900 & 1.03 & 0.95 & 0.90 \\
\hline
\end{tabular}

a Determined by $500 \mathrm{MHz}{ }^{1} \mathrm{H}$ NMR.

b Maximum conversion of macromonomer in the copolymerization with MMA and AIBN in benzene for 48 hours at $60^{\circ} \mathrm{C}$.
PSt were characterized by the same procedure in the previous paper. ${ }^{9}$ Characteristics of the macromonomers and the inert PSts are shown in Table I.

\section{Polymerization of Macromonomers}

Polymerization of the polystyrene macromonomers was carried out in benzene at $60^{\circ} \mathrm{C}$ with $\alpha, \alpha^{\prime}$-azobisisobutylonitrile (AIBN). A mixture of macromonomer, inert PSt, benzene and AIBN was equally divided into sixteen parts and placed in glass ampules. Each ampule was degassed and sealed under vacuum. These ampules were placed in the thermostated bath regulated at $60^{\circ} \mathrm{C}$, and then polymerizations were carried out for various times. All polymerization solutions were homogeneous through out the polymerization reaction. After the polymerization, the ampule was cooled to $-78^{\circ} \mathrm{C}$, and the polymerization product was taken out and freeze-dried with benzene.

\section{Evaluation of Molecular Weight and Polym- erization Rate}

The polymerization rates $\left(R_{\mathrm{p}}\right)$ and the weight average molecular weights of poly(macromonomer)s $\left(M_{w}\right)$ were determined by using a GPC apparatus equipped with a low-angle laser light scattering detector in addition to the conventional IR and UV detectors (LS-GPC). ${ }^{7}$ The GPC apparatus and evaluation procedure of $M_{w}$ of the poly(macromonomer)s were described in the previous papers. ${ }^{7-9}$ The degree of conversion was determined by GPC charts taken with an UV detector using the ratio of the peak area of the polymerization product multiplied by the composition of macromonomer/inert PSt mixture.

\section{ESR Measurements}

ESR spectra were taken at $60^{\circ} \mathrm{C}$ during polymerization in an ESR sample tube. The ESR spectrometer and the procedure of ESR measurements and the determination of the concentration of propagating radicals $\left(\left[\mathrm{M}^{*}\right]\right)$ were described in the previous papers. ${ }^{8,9,15}$ 


\section{RESULTS AND DISCUSSION}

Figure 1 shows the effects of the presence of inert PSt2900 on the relationship between $D P$ of the polymerization product and [M] of MA-PSt2900. The closed symbols represent $D P$ for the polymerization products of MAPSt2900 in the presence of PSt2900, while the open symbols represent $D P$ for that of

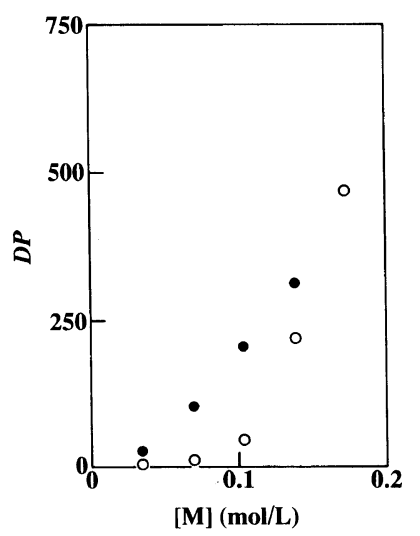

Figure 1. DP of poly(MA-PSt2900) versus $[\mathrm{M}]$ plots in the absence (open symbol) and presence (closed symbol) of PSt2900. PSt 2900 was added to fix the total polymer concentration $([\mathrm{M}]+[\mathrm{PSt}])$ as $1.72 \times 10^{-1} \mathrm{moll}^{-1}$. Polymerizations were carried out in benzene with AIBN at $60^{\circ} \mathrm{C}$ for 24 hours. [I] $=1.6 \times 10^{-2} \mathrm{moll}^{-1}$.

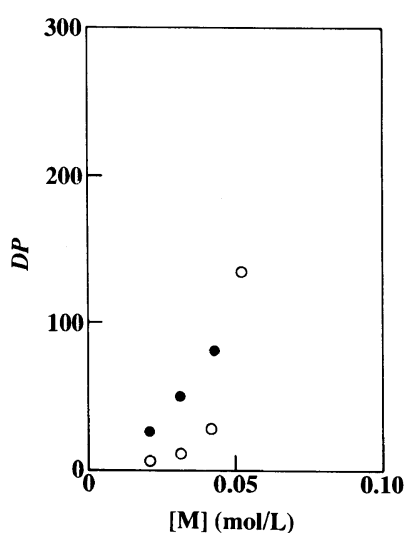

Figure. 2. DP of poly(MA-PSt9600) versus $[\mathrm{M}]$ plots in the absence (open symbol) and presence (closed symbol) of PSt 9400 . PSt 9400 was added to fix the total polymer concentration $([\mathrm{M}]+[\mathrm{PSt}])$ as $5.21 \times 10^{-2} \mathrm{moll}^{-1}$. Polymerizations were carried out in benzene with AIBN at $60^{\circ} \mathrm{C}$ for 24 hours. [I] $=1.6 \times 10^{-2} \mathrm{moll}^{-1}$.
MA-PSt2900 alone. All the polymerizations in the figure were carried out for 24 hours with $[\mathrm{I}]=1.6 \times 10^{-2} \mathrm{moll}^{-1}$. The total polymer concentrations $([\mathrm{M}]+[\mathrm{PSt} 2900])$ in feed are fixed as $1.72 \times 10^{-1} \mathrm{moll}^{-1}$. Thus, the viscosity $(\eta)$ of polymerization media was constant with change of $[\mathrm{M}]$. The same plots for MAPSt9600 are shown in Figure 2, where inert PSt9400 was added to fix the total polymer concentration in feed as $5.21 \times 10^{-2} \mathrm{moll}^{-1}$. It is seen in Figures 1 and 2 that $D P$ of the poly(macromonomer)s increases remarkably with $[\mathrm{M}]$ as in the previous papers. ${ }^{7-9,12}$ Addition of the inert PSt increases $D P$. Variation of $D P$ with [M] in the presence of the inert PSt corresponds to the effect of the concentration of polymerizable end group without the viscosity effect on $D P$.

Figure 3 shows the time-conversion curves for the polymerization of MA-PSt2900 at $[\mathrm{M}]=1.72 \times 10^{-1}$ and $6.90 \times 10^{-2} \mathrm{moll}^{-1}$ and the for MA-PSt2900 with PSt2900 at [M]= $6.90 \times 10^{-2} \mathrm{moll}^{-1}$ and $[\mathrm{PSt}]=1.03 \times 10^{-1}$ $\mathrm{moll}^{-1}\left([\mathrm{M}]+[\mathrm{PSt}]=1.72 \times 10^{-1} \mathrm{moll}^{-1}\right)$. $R_{\mathrm{p}}$ estimated from the initial slope of the time-conversion curve increases with [M]. It is seen that the presence of inert PSt increases $R_{\mathrm{p}}$ at the same [M], but partial replacement

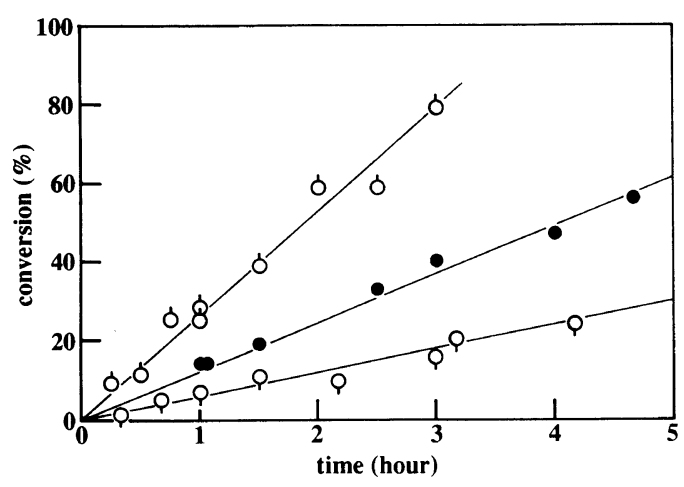

Figure 3. Comparison of time-conversion curves for the polymerization of MA-PSt 2900 at $[\mathrm{M}]=1.72 \times 10^{-1}(\mathrm{O})$ and $6.90 \times 10^{-2}(Q) \mathrm{moll}^{-1}$ and that of MA-PSt2900/ PSt 2900 mixture at $[\mathrm{M}]=6.90 \times 10^{-2} \mathrm{moll}^{-1}$ with $[\mathrm{PSt}]=$ $1.03 \times 10^{-1} \mathrm{moll}^{-1}(\mathbf{O})$. Polymerizations were carried out in benzene with $\mathrm{AIBN}$ at $60^{\circ} \mathrm{C}$. $[\mathrm{I}]=1.6 \times 10^{-2} \mathrm{moll}^{-1}$. 


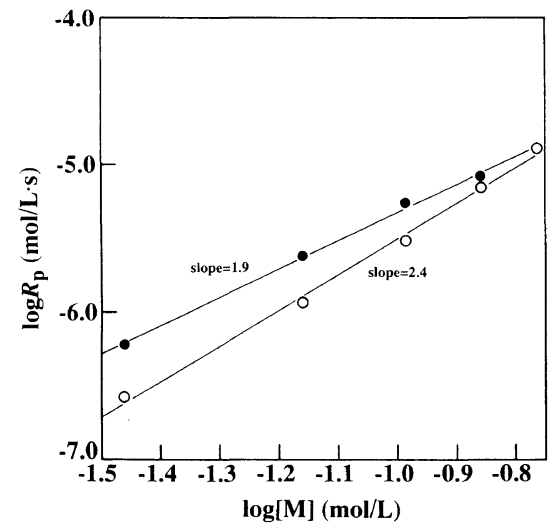

Figure 4. Log-log plots of $R_{\mathrm{p}}$ versus $[\mathrm{M}]$ of MA-PSt 2900 in the absence (open symbol) and presence (closed symbol) of PSt2900. PSt2900 was added to fix the total polymer concentrations $([\mathrm{M}]+[\mathrm{PSt}])$ as $1.72 \times 10^{-1} \mathrm{moll}^{-1}$. Polymerizations were carried out in benzene with AIBN at $60^{\circ} \mathrm{C}$. $[\mathrm{I}]=1.6 \times 10^{-2} \mathrm{moll}^{-1}$.

of the macromonomer with the inert PSt decreases $R_{\mathrm{p}}$.

Figure 4 shows $\log -\log$ plots of $R_{\mathrm{p}}$ versus [M] for the polymerizations of MA-PSt2900 alone and MA-PSt2900 with PSt2900. The presence of PSt2900 increases $R_{\mathrm{p}}$ of MAPSt2900 at the same [M]. This is the result of the decrease of the termination rate with increase of $\eta$ by the addition of the inert PSt. $\log R_{\mathrm{p}}$ of both MA-PSt2900 alone and MA-PSt2900 with PSt2900 increases almost linearly with $\log [\mathrm{M}]$. The apparent kinetic order of $R_{\mathrm{p}}$ with respect to [M] is 2.4 for the macromonomer alone while the order is 1.9 for MA-PSt2900 with PSt2900. The difference of the order, 0.5 , between them is ascribed to the variation of the viscosity effect with $[\mathrm{M}]$.

Figure 5 shows the same $\log -\log$ plots of $R_{\mathrm{p}}$ versus $[\mathrm{M}]$ for the polymerization of $\mathrm{MA}$ PSt9600. In this case, the apparent kinetic order of $R_{\mathrm{p}}$ with respect to [M] is 2.0 for the MA-PSt9600 macromonomer alone and 1.5 for the MA-PSt9600 with PSt9400. Again, the kinetic order decreases 0.5 by the addition of PSt9400.

Propagation rate constant, $k_{\mathfrak{p}}$, and termination rate constant, $k_{t}$, for the radical po-

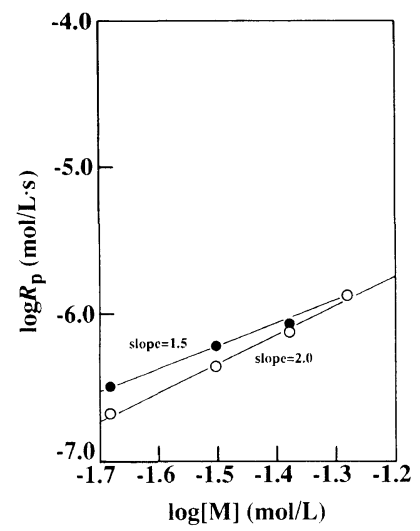

Figure 5. Log-log plots of $R_{\mathrm{p}}$ versus [M] of MA-PSt9600 in the absence (open symbol) and presence (closed symbol) of PSt9400. PSt 9400 was added to fix the total polymer concentrations $([\mathrm{M}]+[\mathrm{PSt}])$ as $5.21 \times 10^{-1} \mathrm{moll}^{-1}$. Polymerizations were carried out in benzene with AIBN at $60^{\circ} \mathrm{C}$. $[\mathrm{I}]=1.6 \times 10^{-2} \mathrm{moll}^{-1}$.

lymerization of the macromonomers are estimated by measurement of $\left[\mathrm{M}^{*}\right]$ with $\mathrm{ESR}$, using eq 1 and $2 .{ }^{15}$

$$
\begin{aligned}
& k_{\mathrm{p}}=R_{\mathrm{p}} /[\mathrm{M}]\left[\mathrm{M}^{*}\right] \\
& k_{\mathrm{t}}=R_{\mathrm{p}} / 2 D P\left[\mathrm{M}^{*}\right]^{2}
\end{aligned}
$$

Equation 2 is based on assumptions of the termination reaction by the disproportionation and no chain transfer reactions to macromonomer, poly(macromonomer) and solvent. The estimated $k_{\mathrm{p}}$ and $k_{\mathrm{t}}$ values are shown in Tables II and III with $R_{\mathrm{p}}, D P$, and [ $\left.\mathrm{M}^{*}\right]$.

In the tables, $\left[\mathrm{M}^{*}\right]$ in the presence of the inert PSt is larger than that in the MA-PSt alone. This indicates that the lifetime of propagating radical becomes longer in the presence of the inert PSt.

It is seen in Tables II and III that $k_{\mathrm{p}}$ is around $25-35\left(1(\mathrm{~mol} \cdot \mathrm{s})^{-1}\right)$. These values are approximately constant and almost independent of [M] and $M_{n}$ of the macromonomer including the results in previous reports. ${ }^{8,9,12,15}$ Thus, $k_{\mathrm{p}}$ is almost constant with change of $\eta$ in this [M] range. Moreover, the inert PSt does not seem to affect the $k_{\mathrm{p}}$ values. The $k_{\mathrm{p}}$ values of the macromonomers are one order smaller than that reported for methyl methacrylate 
Table II. Evaluation of propagation rate constant $\left(k_{\mathrm{p}}\right)$ and termination rate constant $\left(k_{\mathrm{t}}\right)$ for the polymerization of MA-PSt $2900^{a}$ in the absence and presence of PSt 2900

\begin{tabular}{|c|c|c|c|c|c|c|c|}
\hline$[\mathrm{MA}-\mathrm{PSt}]^{\mathrm{b}}$ & {$[\mathrm{PSt}]^{\mathfrak{c}}$} & $R_{\mathrm{p}} \times 10^{6}$ & Dמ & {$\left[\mathrm{M}^{*}\right] \times 10^{6}$} & $k_{\mathrm{p}}^{\mathrm{d}}$ & $k_{\mathrm{t}}^{\mathrm{e}}$ & $f_{f}^{f}$ \\
\hline $\mathrm{moll}^{-1}$ & $\mathrm{moll}^{-1}$ & $\operatorname{mol}(1 \cdot s)^{-1}$ & & $\mathrm{moll}^{-1}$ & $1(\mathrm{~mol} \cdot \mathrm{s})^{-1}$ & $1(\mathrm{~mol} \cdot \mathrm{s})^{-1}$ & \\
\hline 0.172 & 0 & 13 & 470 & 2.6 & 28 & 2000 & 0.084 \\
\hline 0.138 & 0 & 7.0 & 220 & 1.8 & 28 & 4700 & 0.097 \\
\hline 0.103 & 0 & 3.0 & 46 & 0.94 & 31 & 37000 & 0.20 \\
\hline 0.0690 & 0 & 1.2 & 11 & 0.70 & 24 & 100000 & 0.31 \\
\hline 0.0345 & 0 & 0.26 & 3.4 & 0.28 & 20 & 270000 & 0.14 \\
\hline 0.138 & 0.0345 & 8.4 & 310 & 2.0 & 30 & 3300 & 0.081 \\
\hline 0.103 & 0.0690 & 5.5 & 200 & 1.7 & 32 & 4900 & 0.081 \\
\hline 0.0690 & 0.103 & 2.4 & 100 & 1.0 & 34 & 13000 & 0.078 \\
\hline 0.0345 & 0.138 & 0.60 & 28 & 0.75 & 23 & 25000 & 0.084 \\
\hline
\end{tabular}

a Polymerizations were carried out in benzene with AIBN at $60^{\circ} \mathrm{C}$. $[\mathrm{I}]=1.6 \times 10^{-2} \mathrm{moll}^{-1}$.

b Concentration of MA-PSt2900. Not corrected with end functionality.

c Concentration of PSt2900.

${ }^{\mathrm{d}} k_{\mathrm{p}}=R_{\mathrm{p}} /[\mathrm{M}]\left[\mathrm{M}^{*}\right]$.

e $k_{\mathrm{t}}=R_{\mathrm{p}} / 2 D P\left[\mathrm{M}^{*}\right]^{2}$.

f $f=\left(R_{\mathrm{p}} / k_{\mathrm{p}}[\mathrm{M}]\right)^{2} k_{\mathrm{t}} / k_{\mathrm{d}}[\mathrm{I}]$.

Table III. Evaluation of propagation rate constant $\left(k_{\mathrm{p}}\right)$ and termination rate constant $\left(k_{\mathrm{t}}\right)$ for the polymerization of MA-PSt $9600^{\mathrm{a}}$ for the absence and presence of PSt9400

\begin{tabular}{|c|c|c|c|c|c|c|c|}
\hline$[\mathrm{MA}-\mathrm{PSt}]^{\mathrm{b}}$ & {$[\mathrm{PSt}]^{\mathrm{c}}$} & $R_{\mathrm{p}} \times 10^{6}$ & PD & {$\left[\mathrm{M}^{*}\right] \times 10^{6}$} & $k_{\mathrm{p}}^{\mathrm{d}}$ & $k_{\mathrm{t}}^{\mathrm{e}}$ & $f^{\mathrm{f}}$ \\
\hline $\mathrm{moll}^{-1}$ & $\mathrm{moll}^{-1}$ & $\operatorname{mol}(1 \cdot s)^{-1}$ & & $\mathrm{moll}^{-1}$ & $1(\mathrm{~mol} \cdot \mathrm{s})^{-1}$ & $1(\mathrm{~mol} \cdot \mathrm{s})^{-1}$ & \\
\hline 0.0521 & 0 & 1.3 & 130 & 1.1 & 23 & 4200 & 0.030 \\
\hline 0.0417 & 0 & 0.75 & 27 & 0.86 & 21 & 18000 & 0.083 \\
\hline 0.0313 & 0 & 0.43 & 11 & 0.55 & 25 & 66000 & 0.12 \\
\hline 0.0208 & 0 & 0.21 & 5.8 & 0.44 & 23 & 93000 & 0.11 \\
\hline 0.0417 & 0.0104 & 0.98 & 81 & 0.95 & 25 & 6800 & 0.037 \\
\hline 0.0303 & 0.0208 & 0.60 & 50 & 0.74 & 26 & 11000 & 0.036 \\
\hline 0.0208 & 0.0313 & 0.32 & 26 & 0.54 & 29 & 22000 & 0.038 \\
\hline
\end{tabular}

a Polymerizations were carried out in benzene with AIBN at $60^{\circ} \mathrm{C}$. $[\mathrm{I}]=1.6 \times 10^{-2} \mathrm{moll}^{-1}$.

b Concentration of MA-PSt9600. Not corrected with end functionality.

c Concentration of PSt9400.

d $k_{\mathrm{p}}=R_{\mathrm{p}} /[\mathrm{M}]\left[\mathrm{M}^{*}\right]$.

e $k_{\mathrm{t}}=R_{\mathrm{p}} / 2 D P\left[\mathrm{M}^{*}\right]^{2}$.

f $f=\left(R_{\mathrm{p}} / k_{\mathrm{p}}[\mathrm{M}]\right)^{2} k_{\mathrm{t}} / k_{\mathrm{d}}[\mathrm{I}]$.

(MMA). ${ }^{16}$ These results might be explained as that the propagation reaction is not diffusioncontrolled but rather activation-controlled reaction. Small $k_{\mathbf{p}}$ values compared with that of MMA are probably due to the specific multibranched structure in the vicinity of the propagating radical sites.

On the other hand, $k_{\mathrm{t}}$ rapidly decreases from 270000 to $20001(\mathrm{~mol} \cdot \mathrm{s})^{-1}$ for MA-PSt2900 in Table II and from 93000 to $42001(\mathrm{~mol} \cdot \mathrm{s})^{-1}$ for MA-PSt9600 in Table III with increase of [M] without the inert PSt. $k_{\mathrm{t}}$ is also decreased 


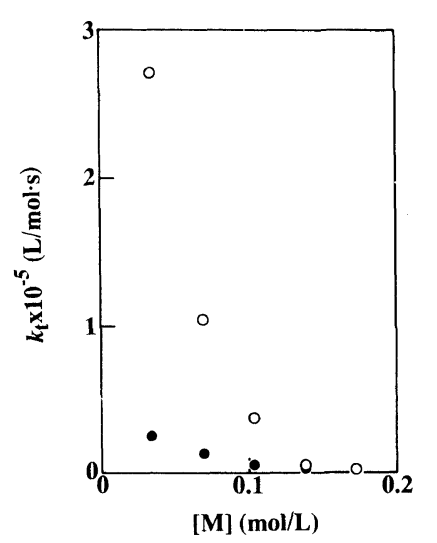

Figure 6. Dependence of $k_{\mathrm{t}}$ on [M] for MA-PSt2900 in the absence (open symbol) and presence (closed symbol) of PSt2900. PSt2900 was added to fix the total polymer concentrations $([\mathrm{M}]+[\mathrm{PSt}])$ as $1.72 \times 10^{-2} \mathrm{moll}^{-1}$. Polymerizations were carried out in benzene with AIBN at $60^{\circ} \mathrm{C}$. [I] $=1.6 \times 10^{-2} \mathrm{moll}^{-1}$.

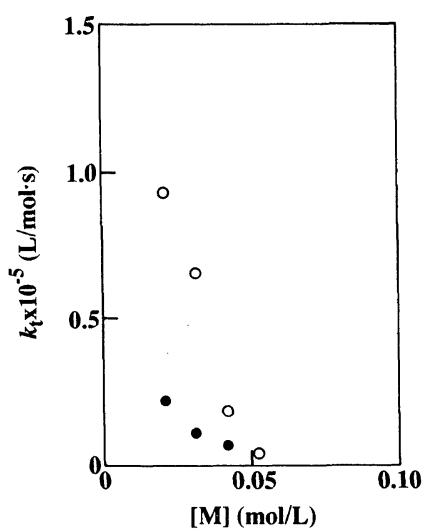

Figure 7. Dependence of $k_{\mathrm{t}}$ on [M] for MA-PSt9600 in the absence (open symbol) and presence (closed symbol) of PSt9400. PSt9400 was added to fix the total polymer concentrations $([\mathrm{M}]+[\mathrm{PSt}])$ as $5.21 \times 10^{-2} \mathrm{moll}^{-1}$. Polymerizations were carried out in benzene with AIBN at $60^{\circ} \mathrm{C}$. $[\mathrm{I}]=1.6 \times 10^{-2} \mathrm{moll}^{-1}$.

by the addition of the inert PSt at the fixed [M]. Moreover, at the nearly same [M], $k_{\mathrm{t}}$ for MA-PSt9600 (Table III) is smaller than that for MA-PSt2900 (Table II). $k_{\mathrm{t}}$ values are plotted against [M] in Figures 6 and 7. These results indicate that the termination reaction is a fast reaction between two propagating radicals and is easily diffusion-controlled, thus sensitive to $\eta$. But, $k_{\mathrm{t}}$ still decreases with increase of $[\mathrm{M}]$ under constant $\eta$, in turn, at a fixed concentration of the sum of the macromonomer and inert PSt. This might indicate that the termination reaction is affected by other factors than the viscosity effect such as the segment density around the active site of propagating radical from the multibranched structure, which depends on $D P$ of the propagating radical. Thus depends on [M].

The effect of the multibranched structure of the propagating radical is also seen by comparing the ESR spectrum of the propagating radical for MA-PSt macromonomer and that of MMA in the presence of inert PSt having the same molecular weight with the macromonomer which corresponds to the system of the polymerizable end group separated from the PSt chain of MA-PSt macromonomer. ${ }^{15}$ From these results, we can safely conclude that the kinetics of the radical polymerization of the PSt macromonomer is affected not only by the viscosity of polymerization media but also the multibranched structure of the propagating radical. Moreover, $D P$ of poly(macromonomer)s did not depend on the conversion of macromonomer or the polymerization time. ${ }^{7,12}$ Thus, the degree of the multibranched structure does not change irrespective of the polymerization time and mainly depends on [M].

Initiator efficiency $(f)$ in Tables II and III was determined by the eq 3 ,

$$
f=\left(R_{\mathrm{p}} / k_{\mathrm{p}}[\mathrm{M}]\right)^{2} k_{\mathrm{t}} / k_{\mathrm{d}}[\mathrm{I}]
$$

$f$ values gradually decrease with increase of $[\mathrm{M}]$ in the absence of the inert PSt. This is the same tendency in the previous paper, ${ }^{9}$ and also seen in the dependence of $f$ on the conversion in the bulk polymerization of $\mathrm{MMA}^{17}$ However, $f$ values are nearly constant in the presence of the inert PSt. These results indicate that $f$ is mainly influenced by $\eta$, irrespective of the concentration of the polymerizable end group under the polymerization conditions in this work. Therefore, the increase in the kinetic 
order larger than 0.5 in $R_{\mathrm{p}}$ with respect to [M] by the addition of the inert PSt might be ascribed to the $[\mathrm{M}]$-dependence of both $\eta$ and $f$.

The termination rate constant $k_{\mathrm{t}}$ is affected by the viscosity and multibranched structure of the propagating radical and given by

$$
\begin{aligned}
k_{\mathrm{t}} & =k_{\mathrm{t} 0} \phi_{\eta} \phi_{\mathrm{mb}} \\
& =k_{\mathrm{t} 0}\left(\eta_{0} / \eta\right) \phi_{\mathrm{mb}}
\end{aligned}
$$

Thereby, $\phi_{\eta}$ and $\phi_{\mathrm{mb}}$ represent influential factors on the viscosity and multibranched structure, respectively. $k_{\mathrm{t} 0}$ is the termination rate constant defined by $k_{\mathrm{t} 0}=k_{\mathrm{t}} \cdot\left(\eta / \eta_{0}\right) . \eta$ is measured viscosity of polymerization media and $\eta_{0}$ is that of the solvent. Thus,

$$
R_{\mathrm{p}} \propto\left(f / k_{\mathrm{t}}\right)^{1 / 2}[\mathrm{M}]=\left(f / k_{\mathrm{t} 0} \eta_{\mathrm{o}} \phi_{\mathrm{mb}}\right)^{1 / 2}[\mathrm{M}] \eta^{1 / 2}
$$

Dependence of $R_{\mathrm{p}}$ on $\eta^{1 / 2}$ was reported by Yokota et al. ${ }^{18}$ Furthermore, the dependence of $f, \phi_{\mathrm{mb}}$ and $\eta$ on [M] may be represented by

$$
\begin{aligned}
f & \propto[\mathrm{M}]^{\beta_{\mathrm{f}}} \\
\phi_{\mathrm{mb}} & \propto[\mathrm{M}]^{\beta_{\mathrm{mb}}} \\
\eta & \propto[\mathrm{M}]^{\beta_{\eta}}
\end{aligned}
$$

$\beta_{\mathrm{f}}, \beta_{\mathrm{mb}}$, and $\beta_{\eta}$ are the exponents of [M] and are negative judging from the experimental data. Thus, the kinetic order of $R_{\mathrm{p}}$ with respect to $[\mathrm{M}]$ is expressed by

$$
R_{\mathrm{p}} \propto[\mathrm{M}]^{1.0}[\mathrm{M}]^{\beta_{\mathrm{f}} / 2}[\mathrm{M}]^{-\beta_{\mathrm{mb} / 2}}[\mathrm{M}]^{\beta_{\eta} / 2}
$$

then

$$
R_{\mathrm{p}} \propto[\mathrm{M}]^{1.0+\alpha_{\eta}+\alpha_{\mathrm{mb}}+\alpha_{\mathrm{f}}}
$$

whereby,

$$
\alpha_{\eta}=\beta_{\eta} / 2, \quad \alpha_{\mathrm{mb}}=-\beta_{\mathrm{mb}} / 2, \quad \alpha_{\mathrm{f}}=\beta_{\mathrm{f}} / 2
$$

In the polymerization of the macromonomer alone, $\alpha_{\eta}, \alpha_{\mathrm{mb}}$, and $\alpha_{\mathrm{f}}$ in eq 4 are not zero. On the other hand, $\eta$ of the polymerization media does not depend on [M] in the polymerization with the inert PSt which leads to $\alpha_{\eta}=0 . \alpha_{\mathrm{f}} \fallingdotseq 0$ which is seen in Tables II and III. Therefore, the additional term in the kinetic order larger than unity corresponds to $\alpha_{\mathrm{mb}}$ for the polymerization with inert PSt. In turn, the multibranched structure does influence the kinetic order of the radical polymerization of the macromonomers. The difference of 0.5 order caused by the addition of inert PSt may correspond to $\alpha_{\eta}+\alpha_{\mathrm{f}}$.

\section{REFERENCES}

1. E. Trommsdorf, H. Kohle, and D. Lagally, Makromol. Chem., 1, 169 (1948).

2. N. Nishimura, J. Macromol. Chem., 1, 257 (1966).

3. D. T. Turner, Macromolecules, 10, 221 (1977).

4. G. Odian, "Principles of Polymerization," 3rd ed., Wiley-Interscience, New York, N.Y., 1991.

5. Y. Tsukahara, in "Macromolecular Design: Concept and Practice," M. Mishra, ed., Polymer Frontiers International Inc., New York, 1994, p 161.

6. I. Capek and M. Akashi, J. Macromol. Sci-Rev. Macromol. Chem. Phys., C33(4), 369 (1993).

7. Y. Tsukahara, K. Mizuno, A. Segawa, and Y. Yamashita, Macromolecules, 22, 1546 (1989).

8. Y. Tsukahara, K. Tsutsumi, Y. Yamashita, and S. Shimada, Macromolecules, 23, 5201 (1990).

9. K. Tsutsumi, Y. Okamoto, and Y. Tsukahara, Polymer, 35, 2205 (1994).

10. R. Asami, M. Takaki, K. Kyuda, and E. Asakura, Polym. J., 15, 139 (1983).

11. R. Asami, M. Takaki, K. Kyuda, and N. Sukenaga, Polym. J., 15, 261 (1983).

12. K. Tsutsumi, Y. Tsukahara, and Y. Okamoto, Polym. J., 26, 13 (1994).

13. K. Ito, K. Tanaka, H. Tanaka, G. Imai, S. Kawaguchi, and S. Itsuno, Macromolecules, 24, 2348 (1991).

14. K. Ito and H. Kobayashi, Polym. J., 24, 199 (1992).

15. Y. Tsukahara, K. Tsutsumi, Y. Yamashita, and S. Shimada, Macromolecules, 22, 2869 (1989).

16. J. Brandrup and E. H. Immergut, Ed., "Polymer Handbook," 3rd ed., Wiley-Interscience, New York, N.Y., 1989, p II/67.

17. G. T. Russell, D. H. Napper, and R. G. Girbert, Macromolecules, 21, 2141 (1988).

18. K. Yokota and M. Ito, Polym. Lett., 6, 825 (1968). 